\title{
MECHANICAL AND THERMODYNAMICAL STUDY OF A MACROSCOPICALLY COHERENT PHASE TRANSITION. CASE OF THE MARTENSITIC TRANSFORMATION
}

\author{
J.F. GANGHOFFER, S. DENIS, E. GAUTIER, A. SIMON, K. SIMONSSON*and S. SJÖSTRÖM* \\ Laboratoire de Science et Génie des Matériaux Métalliques, Ecole des Mines, Parc de Saurupt, \\ F-54042 Nancy cedex, France \\ ${ }^{*}$ Linköping Institute of Technology, S-58183 Linköping, Sweden
}

\begin{abstract}
In the general framework of a macroscopically coherent phase transition, the mechanical and thermodynamical behaviour of a two-phase volume element under structural evolution will be investigated and discussed. The identification of internal entropy production will then allow to formulate a general evolution condition for such a system and the internal stress state will appear to influence strongly the transformation behaviour, via the interface. The case of a martensitic transformation is considered. From that rigourous mechanical approach, we obtain the thermodynamical balance equation used for martensitic transformation.
\end{abstract}

\section{INTRODUCTION}

A great deal of processes involve the propagation of an interface between nonhydrostatically stressed solids, without occurence of diffusion, [1]. The case of macroscopically coherent phase transitions will be investigated here, the coherency condition being associated with the hypothesis of displacement continuity accross the propagating interface. A more thorough discussion of this concept - when considering both atomic and continuum scales - is presented in [1] or [2,3].

The basic hypothesis defining the frame of this study will be presented first. The principle of virtual power is applied in order to investigate the mechanical behaviour of the system considered.

\section{General considerations}

The system under consideration consists of a small closed volume element containing a mixture of two interacting phases, separated by a moving interface. It can be for example a micro-domain contained in a macroscopic sample. Figure 1 provides a description of its accompanying parameters :

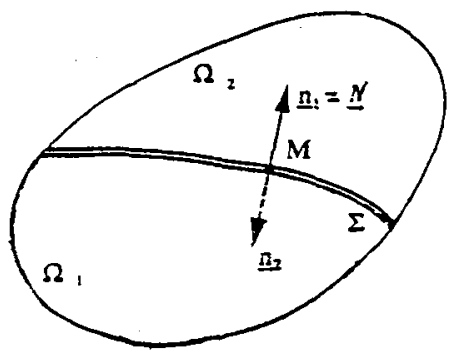

\footnotetext{
$\underline{N}$ is the normal vector at the interface $\Sigma$ indicating its local direction of propagation and $y$ the local velocity of $\Sigma$ at the same point. The interface defines instantaneously a separation between the volumes $\Omega_{1}$ of the new phase and $\Omega_{2}$ of the parent phase. The total volume is denoted $\Omega$.

Since we follow the volume $\Omega$ during its deformation - and transformation-process, the material point of view will be considered here ; to any point $M$ within $\Omega$ is associated a vector $X$ in the reference configuration.
} 
The behaviour of the phases is supposed elastic and the transformation occurs under isothermal conditions. For points $M$ lying on the interface, the coherency condition expresses as $\Delta(\underline{X})=0$ [4], where $\Delta$ is the jump operator $\left[\Delta(\varphi)=\varphi^{(1)_{-}(2)}\right]$ for any physical quantity $\varphi$ experiencing a discontinuity on $\Sigma$. The existence of a moving interface manifests a mathematical and physical link between the behaviour of any quantity defined in the bulk phases and its interfacial behaviour. A first preliminary result of significant use in the following is the expression of the material derivative of the volume integral of a quantity I defined by its volumic density $\mathrm{b}$ in the actual configuration $: I=\int_{\Omega} \mathrm{b}(X, t) d \Omega$, leading to $\frac{\mathrm{dI}}{\mathrm{dt}}=\int_{\Omega} \frac{\partial \mathrm{b}}{\partial \mathrm{t}} \mathrm{d} \Omega+\int_{\partial \Omega} \mathrm{b} \cdot \underline{\mathrm{v}} \mathrm{d} \underline{\mathrm{A}}$, in which $d A$ is the infinitesimal area vector on the boundary $\partial \Omega$, including $\Sigma$ [5]. The first term of the righthand side can be interpreted as the variation of $b$ in the domain $\Omega$ considered as fixed, while the second is the convection due to the variation of $\Omega$. Considering our Lagrangian point of view, this last term will be non zero only at the interface and one has therefore $\frac{d I}{d t}=\int_{\Omega} \frac{\partial b}{\partial t} d \Omega+\int_{\Sigma} \Delta(b) V_{N} d A$, where $V_{N}$ is the normal interfacial velocity.

\section{Mechanical balance}

The principle of virtual power $[6,7]$ receives the general formulation $P(a)=P(e)+P(i)$, where $P(a)$ is the (virtual) power of accelerations, $P(e)$ the power of external forces (acting on the boundaries of the volume element), and $\mathrm{P}(\mathrm{i})$ is the power of internal forces. At this stage, a next hypothesis of quasi-static evolution is formulated, leading to $\mathrm{P}(\mathrm{e})+\mathrm{P}(\mathrm{i})=0$. This means that for constant values of the transformed fraction, no time is needed to reach mechanical equilibrium. The body forces are supposed negligible.

Expression for $P(e)$ is $P(e)=\int_{\partial \Omega} t \cdot \underline{v}^{T} d A$, where $t=\underline{\underline{T}} \cdot \underline{N}$ is the stress vector $(\underline{\underline{T}}$ is the first Piola-Kirchhoff stress tensor, [7]). The surface density of efforts on $\Sigma$ is $\left(\underline{T}_{1} \cdot \underline{N}_{1} \underline{v}_{1} T+\underline{T}_{2} \cdot \underline{N}_{2} \underline{v}_{2} T\right)$ and therefore

$\mathrm{P}(\mathrm{i})=-\int_{\Omega} \underline{T}: \underline{\hat{F}}^{\mathrm{T}} \mathrm{d} \Omega+\int_{\Sigma}\left(\underline{\underline{T}}_{1} \cdot \underline{\underline{N}}_{1} \underline{\underline{v}}_{1} \mathrm{~T}+\underline{\underline{T}}_{2} \cdot \underline{N}_{2} \underline{\underline{v}}_{2} \mathrm{~T}\right) \mathrm{dA}$. Since $\underline{\underline{N}}_{2}=-\underline{N}_{1}=-\underline{\underline{N}}$ (fig. 1) and considering an hyperelastic material for which $\underline{\underline{T}}=\frac{\partial W}{\partial \underline{F}}$ where $W(\underline{F})$ is the strain energy density $\underline{\underline{F}}$ is the deformation gradient tensor), previous expression of $\mathrm{P(i)}$ can be rewritten as

$\mathrm{P}(\mathrm{i})=-\int_{\Omega} \frac{\partial \mathrm{W}}{\partial \mathrm{t}} \mathrm{d} \Omega+\int_{\Sigma} \Delta\left(\underline{T} \cdot \underline{\underline{N}} \cdot \underline{v}^{\mathrm{T}}\right) \mathrm{dA}$, where $\underline{\mathrm{N}}$ is common to both phases.

Introducing now Hadamard's compatibility condition [4] $\Delta \underline{\mathrm{V}}=-\Delta \underline{\underline{F}} \underline{\mathrm{N}}) \mathrm{V}_{\mathrm{N}}$ in the expression of $\mathrm{P}(\mathrm{i})$ and rewriting equilibrium equation yields

$$
\int_{\partial \Omega} \underline{t} \underline{\underline{v}}^{\mathrm{T}} \mathrm{dA}=\int_{\Omega} \frac{\partial \mathrm{W}}{\partial \mathrm{t}} \mathrm{d} \Omega+\int_{\Sigma} \Delta\left(\underline{N}^{\mathrm{T}} \underline{\underline{F}}^{\mathrm{T}} \underline{\underline{T}} \underline{\mathrm{N}}\right) \mathrm{V}_{\mathrm{N}} \mathrm{dA}
$$

Since a quasi-static hypothesis is assumed, we can substitute the dependence on the transformed fraction $x$ to the dependence on time (time piays here the role of an history parameter), obtaining thereby :

$$
\int_{\partial \Omega} \underline{t} \cdot \frac{d \underline{u} T}{d x} d A=\int_{\Omega_{1} U \Omega_{2}} \frac{\partial W}{\partial x} d \Omega+\int_{\Sigma} \Delta\left(\underline{N}^{T} \cdot \underline{F}^{T} \underline{T} \cdot \underline{N}\right) \frac{d r_{N}}{d x} d A
$$

considering a nearby uniform interfacial velocity . 
The term $\int_{\Omega_{1} \mathrm{U} \Omega_{2}} \frac{\partial \mathrm{W}}{\partial \mathrm{x}} \mathrm{d} \Omega$ represents the variations of strain energy of each phases, due to a $\mathrm{dx}$ and will be denoted $\frac{d E^{e}}{d x}(1)+\frac{d E^{e}}{d x}(2)$. The interfacial term expresses itself as $\frac{\Omega}{\Sigma} \int_{\Sigma} \Delta\left(\underline{\underline{N}}^{\mathrm{T}} \cdot \underline{\underline{F}}^{\mathrm{T}} . \underline{\underline{\mathrm{T}}} \cdot \underline{\mathrm{N}}\right) \mathrm{dA}$. It can be shown that the integrand can be

identified with the jump of $\mathrm{W}$ at equilibrium and last integral receives therefore the more simple expression

$\underline{\Sigma} \int_{\Sigma} \Delta(\mathrm{W}) \mathrm{dA}=\Omega \cdot \overline{\Delta(\mathrm{W})}$, where $\Delta(\mathrm{W})=\Delta\left(\underline{\mathrm{N}}^{\mathrm{T}} \cdot \underline{\underline{\mathrm{F}}} \mathrm{T} \cdot \underline{\underline{T}} \cdot \underline{\mathrm{N}}\right)$ is averaged over the interface. Defining also averaged strain energy in each phase by $\frac{\overline{\mathrm{E}^{\mathrm{e}}}}{\mathrm{dx}}(1)=\frac{1}{\Omega_{1}} \frac{\mathrm{dE}^{\mathrm{e}}}{\mathrm{dx}}(1)$ and $\frac{\mathrm{dE}^{\mathrm{e}}}{\mathrm{dx}}(2)=\frac{1}{\Omega_{2}} \cdot \frac{\mathrm{dE}^{\mathrm{e}}}{\mathrm{dx}}(2)$ provides a more explicit form of (2):

$$
\frac{1}{\Omega} \int_{\partial \Omega} \underline{t} \cdot d \underline{u} \mathrm{~T} d A=x \cdot \widetilde{d E^{e}}(1)+(1-x) \cdot \overline{d E^{e}}(2)+\overline{\Delta(W)} d x
$$

\section{Discussion of the mechanical balance-case of a martensitic transition}

Considering general balance equation (3) makes clearly appear the interaction between the transformation process and the appending mechanical behaviour. The effect of transformation on mechanics is evident when considering the rate form of this equation, an increase of the transformed fraction $\mathrm{dx}$ leading to according variations of the different mechanical contributions it contains.

The two first terms on the right-hand side describe the effect of the transformation process on the variations of strain energies in the bulk phases. The mixture law form evidences how the deformation energy of the mixture will be distributed among each phase, along the transformation. The development of strain energy in each phases, which has both geometrical (there is a continuous change of the volume of each phase) and mechanical origins (build-up of stresses to accommodate the lattice change), should also consider the possible change of elastic properties of a material point. It is likely to have a strong effect on the transformation progress, considering the high deformation energy needed to accommodate the possible large transformation strain (e.g., martensitic transformation is usually associated to a very large shear strain, about 20\%).

The other effect of mechanical behaviour on transformation is mostly evidenced by the term $\overline{\Delta(W)}$, originating at the interface, a material point experiencing a jump of the normal component of tensor $\underline{\underline{F}}^{\mathrm{T}}$. $\underline{\underline{T}}$ when swepped up by the front. At the same time, the normal direction indicates also the local direction of propagation. The stress state appears therefore to control both progress and anisotropy of the transformation, a feature that appears therefore to be of high significance for such non hydrostatically stressed solids. $\overline{\Delta(\mathrm{W})}$ has a driving effect on the transformation and can be therefore called the mechanical driving force. It appears in (3) in a general form and its characterisation for any particular transition needs the formulation of some hypothesis concerning the kinematics of the transformation.

\section{Specification of the mechanical balance for martensitic transformations}

Considered as a mode of deformation, martensitic transformation involves the existence of an invariant plane, usually called habit plane [8]. The deformation associated to the transformation consists in a shear yo along the habit plane and a dilatation $\varepsilon$ normal to it. Such a kinematical description is among the basic features of phenomenological theories [9]. When considering our micro scale approach, what is then needed is a link between both scales, which is found when considering implications of conservation of momentum for a point $M$ at the interface : 
$\Delta(\underline{\mathrm{t}}(\underline{N}))=\mathrm{m} \Delta(\underline{v})$, in which $\underline{\mathrm{v}}$ is the velocity of matter relative to $\Sigma$ and $\mathrm{m}$ the mass flux accross $\Sigma$. Since no diffusion occurs, no mass flux accross the interface can be denoted, leading to the continuity of the stress vector on $\Sigma$ (all components $\mathrm{T}_{\mathrm{iN}}, \mathrm{i}=1,3$ are continuous).

Complementary continuity conditions of the deformation gradient tensor prevail also, as expressed by the

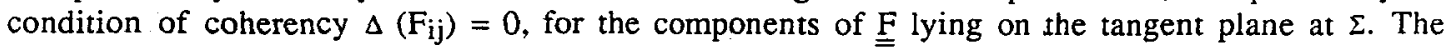
discontinuous part of $\underline{\underline{F}}$ defines the transformation "strain", which can be expressed as a combination of a dilatation عo normal to the tangent plane at $\Sigma$ and a shear yo along some suitable direction $\underline{\mathrm{d}}$ lying on the tangent plane. In the local basis $(d, \underline{N})$, one has therefore $\underline{F}^{t r}(\underline{d}, \underline{N})=\left(\begin{array}{ll}1 & \gamma_{0} \\ 0 & \varepsilon_{0}\end{array}\right)$. Identification of the tangent plane with the concept of habit plane provides then the needed link between micro and raacro scale descriptions.

Evaluation of $\Delta(\mathrm{W})$ requires further to consider the mechanical path (since the transformation involves

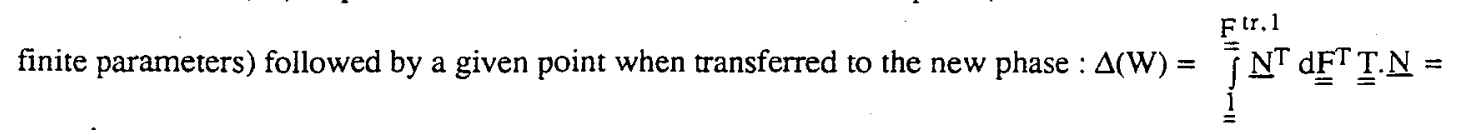
$F^{\text {tr, } 1}$

$\int_{\underline{1}}^{=} \underline{\mathrm{I}}^{\mathrm{T}} \cdot \mathrm{dF}=\underline{\mathrm{N}}$, integrating from the non deformed configuration ( $\underline{\underline{F}}=\underline{\underline{1}}$ ) to the "transformed " one .

Supposing that the continuous stress components don't change during the transformation path (fig. 2) and

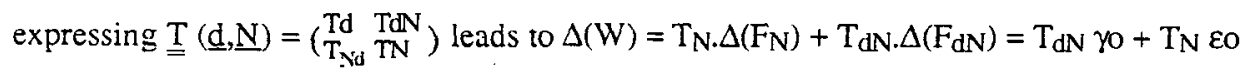

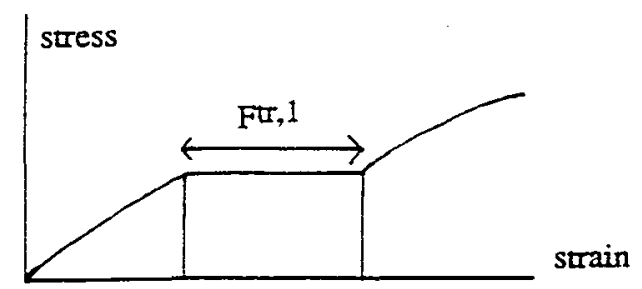

Figure 2: Mechanical path during the transformation.

Equation (3) receives then a more explicit form, still valid for any coherent phase transition :

$$
\frac{1}{\Omega} \int_{\partial \Omega} \underline{t} \cdot d \underline{\underline{u}} \mathrm{~T} d A=x \cdot \overline{\mathrm{dE}^{\mathrm{e}}}(1)+(1-\mathrm{x}) \cdot \overline{\mathrm{dE}^{\mathrm{e}}}(2)+\left(\overline{\mathrm{T}}_{\mathrm{dN}} \gamma_{\mathrm{o}}+\overline{\mathrm{T}}_{\mathrm{N}} \varepsilon o\right) \mathrm{dx}
$$

showing the influence of averaged (on the interface) shear and dilatation components

$\overline{\mathrm{T}}_{\mathrm{dN}}=\frac{1}{\Sigma} \int_{\Sigma} \mathrm{T}_{\mathrm{dN}} \mathrm{dA}$ and $\overline{\mathrm{T}}_{\mathrm{N}}=\frac{1}{\Sigma} \int_{\Sigma} \mathrm{T}_{\mathrm{N}} \mathrm{dA}$ of the stress tensor on the anisotropic interfacial behaviour.

Considering now the hypothesis leading to (4) that could put restrictions on application of present treatment to any kind of macroscopically coherent phase transition, the strongest is that of quasi-static behaviour, since e.g. martensitic transitions occur generally with high interfacial velocity and could need a dynamical treatment instead.

\section{Thermodynamical study of a macroscopically coherent phase transition}

A pure mechanical treatment as detailed before has to be enlarged - via thermodynamics - to integrate all physical entities entering in the transformation process description. The role of chemical energy, essentiel in phase transitions, doesn't apparently enter in previous mechanical approach. Nevertheless, a basic problem arises when considering a definition of chemical potential for two mutually interacting solid phases. If, for fluids, a concept of chemical potential can be introduced naturally - as an indicator of the direction of matter flow - the situation is more complex when considering solid phases for wich nondydrostatic stress states 
may govern the transformation process itself. Therefore, our approach will instead consist in deriving a generalised concept of chemical potential for two evoluting solid phases, from the identification of internal entropy production.

The combination of first and second principle (Lagrangian formalism) yields following expression for the internal entropy production, in the isothermal and quasi-static case :

$$
\frac{\mathrm{d} S \mathrm{i}}{\mathrm{dt}}=\frac{1}{\mathrm{~T}}\left[\int_{\partial \Omega} \mathrm{t} \cdot \underline{v}^{\mathrm{T}} \mathrm{dA}-\frac{\mathrm{d}}{\mathrm{dt}} \int_{\Omega}(\mathrm{u}-\mathrm{Ts}) \mathrm{d} \Omega\right]
$$

For an isothermal process and considering a one phase component, Helmholtz energy density $\psi=f\left(\mathrm{U}-\mathrm{T}_{\mathrm{S}}\right)$ can be identified with strain energy density, [10]. For a two phase component which content of each phase varies, one must also consider the difference of atomic structure between the two phases, involving an additional chemical energy term. $\psi$ can therefore be written as $\psi=W(\underline{\underline{F}})+\mu$, where $\mu$ is the chemical potential of either phase.

Evaluation of quantity $\quad \frac{\mathrm{d}}{\mathrm{dt}} \int_{\Omega} \mathrm{W}(\underline{\mathrm{F}}) \mathrm{d} \Omega=\int_{\Omega} \frac{\partial \mathrm{W}}{\partial \mathrm{F}}: \dot{\underline{F}}^{\mathrm{T}} \mathrm{d} \Omega+\int_{\Sigma} \Delta(\mathrm{W}) \cdot \underline{\mathrm{V}} \cdot \underline{\mathrm{N}} \mathrm{dA}$

can be done using divergence theorem and Hadamard's condition, leading to

$$
\frac{\mathrm{d}}{\mathrm{dt}} \int_{\Omega} \mathrm{W}\left(\underline{\underline{F})} \mathrm{d} \Omega=\int_{\partial \Omega} \underline{\underline{t}} \underline{\underline{y}}^{\mathrm{T}} \mathrm{dA}+\int_{\Sigma} \Delta\left(\mathrm{P}_{\mathrm{NN}}\right) \mathrm{V}_{\mathrm{N}} \mathrm{dA}\right.
$$

in which $P_{N N}$ is the normal component of Eshelby's tensor $\underline{\underline{P}}=\mathrm{W} \underline{\underline{1}}-\underline{F}^{\mathrm{T}}$.T, which plays an important role in the theory of defects in solids $[11,12]$. Since the chemical potentials don't depend on time and are uniform within each phase, one has $\frac{\mathrm{d}}{\mathrm{dt}} \int_{\Omega} \mu \mathrm{d} \Omega=\int_{\Sigma} \Delta(\mu) \mathrm{V}_{\mathrm{N}} \mathrm{dA}=\Delta(\mu) \int_{\Sigma} \mathrm{V}_{\mathrm{N}} \mathrm{dA}=\Delta(\mu) \Omega \frac{\mathrm{dx}}{\mathrm{dt}}$, where $\Delta(\mu)=\mu_{\mathrm{m}}-\mu_{\mathrm{a}}$ is the difference of chemical potentials between both phases. Its introduction in (5)

combined with (7) leads to $\frac{\mathrm{dSi}}{\mathrm{dt}}=-\frac{1}{\mathrm{~T}}\left(\int_{\Sigma} \Delta\left(\mathrm{P}_{\mathrm{NN}}\right) \mathrm{V}_{\mathrm{N}} \mathrm{dA}+\Delta(\mu) \cdot \Omega \frac{\mathrm{dx}}{\mathrm{dt}}\right)$

The irreversible behaviour- if any - has therefore two distinct sources. The mechanical dissipation described by the first term on the right-hand side is located only at the interface, which is logical since the behaviour of the phases is elastic only. A non zero value of this term would imply the existence of latent heat or dissipation by friction at the interface. The chemical term manifests the higher stability of the newly formed phase [13] (the ability for the transformation to occur is related to a negative value of the difference $\left(\mu_{\mathrm{m}}-\mu_{\mathrm{a}}\right)$ for an unstressed volume element ; it is a function of the temperature). Since it arises also from the

interface, (8) can be condensed into $\frac{\mathrm{dSi}}{\mathrm{dt}}=-\frac{1}{\mathrm{~T}} \int_{\Sigma} \Delta(\Sigma \mathrm{NN}) \mathrm{VN} \mathrm{dA}$ (9),

in which $\underline{\underline{\Sigma}}=\underline{\underline{\mathrm{P}}}+\mu \underline{\underline{1}}$ is the energy-momentum tensor [4], which provides a generalised definition of a chemical potential for such non hydrostatically stressed solids. From the positive character of the internal entropy production and considering the arbitrary choice of volume element $\Omega$, one can further deduce a local evolution condition from (9), namely $\Delta\left(\Sigma_{\mathrm{NN}}\right) \leq 0$, which interprets $-\Delta\left(\Sigma_{\mathrm{NN}}\right)$ as a thermodynamical force for the transformation. Introducing now equations $(6)$ and $(7)$ in the expression of $\frac{\mathrm{dSi}}{\mathrm{dt}}$ allows to explicit the evolution conditon as

$$
\int_{\Omega} \frac{\partial \mathrm{W}}{\partial \mathrm{t}} \mathrm{d} \Omega+\int_{\Sigma} \Delta\left(\underline{\underline{N}}^{\mathrm{T}} \underline{\underline{F}}^{\mathrm{T}} \cdot \underline{\underline{\mathrm{T}}} \cdot \underline{\mathrm{N}}\right) \mathrm{V}_{\mathrm{N}} \mathrm{dA}+\Delta(\mu) \cdot \Omega \frac{\mathrm{dx}}{\mathrm{dt}} \leq \frac{1}{\Omega} \int_{\partial \Omega} \underline{\mathrm{t}} \cdot \underline{\underline{v}}^{\mathrm{T}} \mathrm{dA}
$$

equality describing equilibrium.

Comparing now thermodynamical equilibrium equation with the mechanical balance (1) shows that the chemical energy term appears as an additional driving energy for the transformation, which itself results in a storage of strain energy in the bulk phases and - possibly - dissipation at the interface . 
Condition (10) can be equivalently expressed in the $\mathrm{x}$ variable, namely

$$
\Delta(\mu) \mathrm{dx}+\mathrm{x} \overline{\mathrm{dE}^{\mathrm{e}}}(1)+(1-\mathrm{x}) \cdot \mathrm{d} \bar{E}^{\mathrm{e}}(2)+\left(\overline{\mathrm{T}}_{\mathrm{dN}} \gamma \mathrm{o}+\overline{\mathrm{T}}_{\mathrm{N}} \varepsilon \mathrm{o}\right) \mathrm{dx} . \leq \frac{1}{\Omega} \int_{\partial \Omega} \underline{\mathrm{t}} \cdot \mathrm{du} \mathrm{T} \mathrm{dA}
$$

That equation is similar to the one written generally for a transformation under stress, $[13,14]$. The first term represents the difference in chemical energy, the second and third terms deformation energy and the last one the energy associated to the transformation. The energy associated to the creation of a new interface has been neglected, considering a constant area of the moving front.

\section{Formulation of criteria for variants selection in case of a martensitic transformation}

The previous approach shows that both kinetics and anisotropy of the transformation are governed by the coupled influence of deformation energies of the phases and the stress state at the interface. For a martensitic transformation, a F.E. simulation has been recently developed and presented in $[15,16]$. The transformation proceeds by the successive formation of plates within a grain and the local stress state is supposed to control the activation start of each plate : considering a small "nucleation point" for which equation (11) applies, the orientation of the transformation plate is determined by the direction in space (habit plane normal) providing the highest value of expression $\overline{\mathrm{T}}_{\mathrm{dN}} \gamma_{\mathrm{O}}+\overline{\mathrm{T}}_{\mathrm{N}}$ عo. As a first attempt, the role of deformation energies of the phases is neglected in this model. This seems reasonnable when considering only the very first stage of the transformation. However, as for our material, the behaviour of the phases is elastoplastic, additionnal irreversible strain energies enter into account, which varies with the progress of the transformation. Taking it into account needs further developpements both in the thermodynamic approach and in the simulation.

\section{ACKNOWLEDGEMENTS}

The work described has been supported jointly by C.N.R.S. (the French Centre for Scientific Research) and STU (the Swedish Board for Technical Development).

\section{References}

/1/ R.C. FLETCHER - 1973, J. of Geophysical Research, Vol. 78, N³2, 7661.

12/ G.B. OLSON, M. COHEN - 1978, Acta Met., Vol. 27, 1987

13/ F. LARCHE, J.W. CAHN

1978, Acta Met., Vol. 29, 1579

1979, Acta Met., Vol. 26, 53.

14/ W. HEIDUG, F.K. LEHNER - 1985, Pageoph, Vol. 123, 91.

15/ P. GERMAIN - Mécanique des milieux continus, Masson 1986.

16/ J. LEMAITRE, J.L. CHABOCHE - Mécanique des matériaux solides - Dunod.

n) L.E. MALVERN - Introduction to the mechanics of a continuum medium, Prentice-Hall, Inc.

18/ E. PATOOR - Thèse de Docteur-Ingénieur, Université de Metz (1986).

191 M.S. WECHSLER, D.S. LIEBERMAN, T.A. READ

1953, Trans. AIME, 197, 1503.

/10/ M. KATO, H.A. PAK - 1984, Phys. Stat. Sol. (b) 123, 415.

/11/ M. EPSTEIN, G.A. MAUGIN - 1990, Acta Mech. 83, 127-133.

/12/ P. CHADWICK - 1975, J. of Elasticity, vol 5, $\mathrm{N}^{\circ} 3-4$.

113/ J.W. CHRISTIAN - The theory of transformations in Metals and Alloys .Part 1 .(second edition)

114) E.GAUTIER, A.SIMON, X.M. ZHANG, Proceedings "The Martensitic Transformation in Science and Technology,Ed. E.Hornbogen, N.Jost, DGM, 451,1989.

/15/ J.F. GANGHOFFER, S. DENIS, E. GAUTIER, A. SIMON, K. SIMONSSON, S. SJÖSTRÖM, Proceedings ICRS3, 1991.

/16/ J.F. GANGHOFFER, S. DENIS, E. GAUTIER, A. SIMON, K. SIMONSSON, S. SJÖSTRÖM, European Symposium "Martensitic transformation and shape memory properties" Aussois 1991. 